\title{
Influence of Legibility on Perceived Safety in a Virtual Human-Robot Path Crossing Task
}

\author{
Christina Lichtenthäler*, Tamara Lorenz ${ }^{\dagger} \ddagger$, Alexandra Kirsch ${ }^{\S}$
}

\begin{abstract}
In the future robots will more and more enter our daily life. If we want to increase their acceptance it is necessary that people feel safe in the surrounding of robots. As a prerequisite we think that the robot's behavior has to be legible in order to achieve such a feeling of perceived safety. With our present experiment we assess the perceived safety participants feel when an autonomous robot is crossing their path. Therefore participants are presented with a video based scenario in first person perspective. The robot is moving with two different navigation algorithms which allows us to test whether the legibility has an influence on the perceived safety and whether the two navigation algorithms differ regarding their resulting legibility and thus perceived safety. Results show that legibility as defined here increases perceived safety of both navigation methods while the level of perceived safety differs between them.
\end{abstract}

\section{INTRODUCTION}

In the near future not only vacuum cleaner robots will serve in our homes, robots will also help us doing the housework. For a successful human-robot interaction in this context, the user acceptance towards a robotic system plays an important role. Only if the robot is accepted, it will be used. Heerink et al. [1] describes the Almere model of robot acceptance, which is an extension of the UTAUT model [2]. According to Heerink et al., the robot acceptance is influenced by trust, anxiety, perceived usefulness and perceived enjoyment. However, we think, that another important issue of acceptance is the perceived safety. Following Maslow's theory of human motivation [3], safety is a basic human need. Furthermore, Bartneck et al. [4] designate it as a key requirement of HRI and define the perceived safety as follows: Perceived Safety describes the user's perception of the level of danger when interacting with a robot, and the user's level of comfort during the interaction.

According to this definition, perceived safety depends on the ability to perceived one's surrounding and judge the level of immediate danger. Also the factors mentioned by Heerink et al. [1] depend in part on the ability to infer the actions of the robot. Imagine a robot performing some household chore, such as preparing a meal. The robot fulfills its duty, but manipulates objects with sudden, unpredictable movements. It rushes through the kitchen with rapid changes of direction or ignores obvious errors like a pot not being placed properly on the stove. Even if this robot will eventually serve the meal, a person might not have enough trust in the robot to leave it alone, because its actions are neither understandable nor

*Institute for Advanced Study, Technische Universität München †Experimental Psychology, Ludwig-Maximilians-University München $\ddagger$ Automatic Control Engineering (LSR), Technische Universität München $\S$ Department of Computer Science, Tübingen University predictable. How safe would a person feel when the robot's behavior and path are absolutely unpredictable? Probably not very much.

Thus, if perceived safety shall be increased, the behavior of the robot must be predictable in order to react appropriately and in time. Following this argumentation we suggest that the level of perceived safety will increase with the legibility of the robot's behavior, which we define as follows: A robot's behavior is legible, if a human can predict the next actions of the robot and the robot behavior fulfills the expectations of a human interaction partner.

The first aim of this paper is to test, whether the legibility of a robot's behavior increases the perceived safety during interaction with a robot. However, neither the Almere model [1] nor the UTAUT model [2] explicitly contain perceived safety as an acceptance factor. Only Bartneck et al. [4] proposed to measure the perceived safety as a dimension of the user's perception. In his Godspeed $V$ questionnaire participants are asked to rate their emotional state on a 5point semantic differential scale regarding anxious-relaxed, agitated-calm, and quiescent-surprised. Combining the ideas of Bartneck et al. with the fact that safety is a basic need we think that perceived safety - and thus legibility - are basic factors if we want to increase the acceptance of an autonomous robot's behavior.

In this context, a wide range of Wizard of $\mathrm{Oz}$ experiments have been executed to evaluate complex robot behavior, see for example [5], [6], [7], [8]. However, in all experiments of the mentioned studies, behavior is caused by a human operator and not by an implemented algorithm. When talking about autonomous robot behavior though, one is talking about the algorithms that cause the behavior. Therefore, if we want to find out how safety is perceived during interaction with an autonomous robot, it is necessary to directly evaluate the algorithms that cause the behavior. Thinking again about the robot that is rushing through the kitchen, one might be interested in the legibility of the underlying navigation algorithms. Therefore, the second purpose of our experiment is to compare human reactions caused by their perceptions of an autonomous robot whose navigation is steered by different algorithms in a path crossing scenario. Overall the question is whether the behavior of the robot caused by a certain navigation algorithm is legible and if a legible robot behavior increases perceived safety.

In the remainder of this paper we first state how our approach fits the context of related research (section II). Afterwards we introduce our experimental design (section III) and present the results (section IV), which are discussed 
in section V. Finally we conclude and provide an outlook for future work (section VI).

\section{RELATED WORK}

There is only a small number of contributions in the literature dealing with the legibility of robot behavior. Nevertheless there is the wide field of intention recognition, e.g. [9]. Contrary to the task of predicting the intentions of an agent - whether it is a human or a robot - with our study we measure if the behavior of the robot is legible.

Only Takayama et al. [10] we find a comparable experiment towards the legibility (in their work called readability) of the robot behavior. They present a simulation-based experiment to verify the legibility of robot behavior and find support for their hypothesis that the legibility is influenced by the robot showing forethought and goal-oriented reactions. Oppoite to Takayama et al. our experiment is placed in a real world environment with which we measure the legibility of the robot's behavior. But with the study at hand we also want to compare different navigation algorithms. Akin to our comparison are Kulic and Croft [11] who compare two different motion planners with regard to the anxiety of participants in a pick and place task. They compared a classical potential field planner and a safe planner while motion was presented in three different velocities. Here, participants reported less anxiety when safely planned motions were presented at fast speed. For slow and normal speed a significant difference between planners could not be shown. In our study we will also test the anxiety of people interacting with a robot, but unlike Kulic and Croft we treat anxiety only as one item among others leading to the cumulative measure of perceived safety, as proposed by Bartneck et al. [4].

As our interaction task is a path crossing scenario, the work of Dautenhahn et al. [8] also plays a role. Here, an experiment was accomplished in which three different approach strategies were compared with the objective to illuminate preferences. In the study, most participants preferred to be approached from either the left or right side, with a small overall preference for a right side approach by the robot. With the results at hand different approach methods were developed, taking into account the preferred approach directions. Inspired by this work we also included different approach directions in order to control for possible implications.

\section{EXPERIMENTAL DESIGN}

The experiment was designed to address the question of how legible different navigation algorithms are to a human and how this legibility increases the perceived safety during human-robot path crossing. Furthermore we want to answer the question of how legibility and perceived safety during human-robot path crossing are correlated to each other.

\section{A. Platform and Tracking System}

The platform used in this experiment is the omnidirectional RWI B21 robot with a four wheel synchronous drive (see Fig. 2a). The robot has a height of $1.22 \mathrm{~m}$ and a footprint

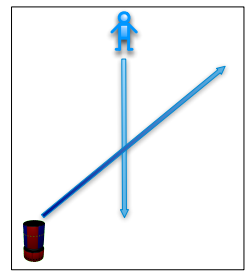

(a)

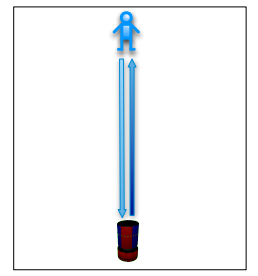

(b)

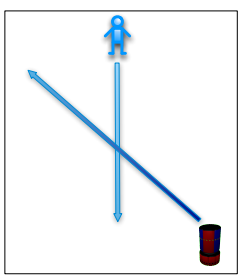

(c)
Fig. 1: Approach directions of the robot (a) from the left, (b) frontal and (c) from the right.

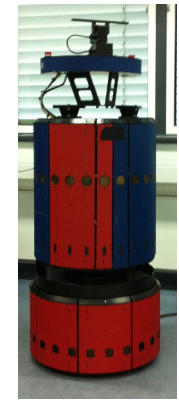

(a)

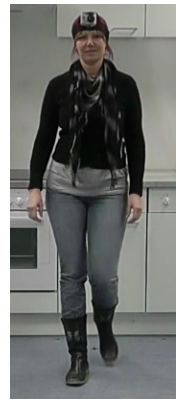

(b)

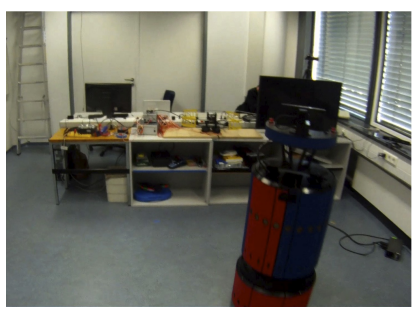

(c)
Fig. 2: Supplementary material and environment for video recording: (a) B21 RWI robot, (b) interactor with head mounted camera, (c) lab area with Kinect sensor as seen from the head mounted camera.

of $0.53 \mathrm{~m}$ in diameter. For localization and navigation it has a SICK laser scanner. For accessing the position and direction of the human interaction partner we utilized a fixed Kinect sensor, which was installed in the lab environment.

Basili et al. [12] showed that gaze has a significant influence on participants' ability to infer the direction of an approaching person or robot. However, in the study at hand we decided to avoid the potentially directing effect of the robot's gaze in order to find out whether and to what extend the behavior of an autonomous agent is legible if the only available cue is its motion. Therefore we choose the B21 robot without any artificial head or eyes, allowing us to concentrate on the legibility of the robot's navigation behavior.

\section{B. Navigation Methods}

In our study we compare the human-aware navigation of Kruse et al. [13] with the state of the art navigation method taken from the ROS navigation stack (www.ros.org/wiki/navigation). These two navigation algorithms entailed the best results in a pilot study [14]. In this section we give a brief introduction to the navigation methods and the differences between them. Both methods consist of a global and a local planner. Global planners generate a complete path to a defined goal pose using an $\mathrm{A}^{*}$ algorithm. The local planner is seeded by the global plan and generates velocity commands to control the robot. Differences between 
global planners result from the cost function the $\mathrm{A}^{*}$ algorithm uses to compute the optimal path, whereas the local planners differ in methods to determine velocity commands. In the following we describe the different global planner cost functions and the different local planners.

a) Move Base Global Planner: The cost function of the move base global planner (MB) is based on a $3 \mathrm{D}$ voxel grid. An obstacle causes infinite costs with descending costs in its surrounding to propagate them from obstacles out to a user-specified radius. For further information see [15] and http://www.ros.org/wiki/navfn.

b) Dynamic Window Approach: The dynamic window approach (DWA) is a real-time collision avoidance strategy developed by Fox et al. [16] (www.ros.org/wiki/dwa_local_planner). The DWA computes local controls by first determining a target trajectory in position or velocity space (usually a circular arc or another simple curve). It then inverts the robot's dynamics to find the desired velocity command that will produce that trajectory [17].

c) Human Aware Global Planner: The cost function of the human aware global planner (HA) takes the human, his/her motions and all obstacles into account. In addition to the infinite cost of obstacles it increases the cost around a human and differentiates between a standing and a moving person:

- Moving: higher cost in front of the human to avoid moving in his/her direction of motion.

- Standing: higher cost behind the human to avoid moving behind his/her back.

Those "social costs" shall ensure a comfortable navigation behavior. For further details see [13].

d) Waypoint Follower Local Planner: The waypoint follower (WF) was developed to execute plans generated by the human-aware global planner. This local planner projects its own motion and the human motion into the future and selects a speed that avoids predictable collisions. The human motion is predicted linearly assuming constant speed and direction, while the robot motion is predicted using the global path returned by the human aware global planner.

\section{Video Clips}

For preparation of the experiment we recorded short video sequences in which a robot is crossing a person's path in our lab environment, see Fig. 1. We used a head mounted goPro camera to provide videos in a first person perspective. In order to guarantee an unbiased interaction, the interactor for video recording was a person not professionally involved in developing navigation algorithms. In all video clips the human interactor starts from one defined position while the start position of the robot was varied, see Fig 1. The interactor starts walking when the robot starts moving.

For the experiment we divided the video into two parts. In the beginning of the video clips both navigation methods (MB-DWA and HA-WF) produce the same robot navigation behavior. The video was cut at the point where the navigation methods are starting to differ in their behavior. When the the robot perceived the human either as an obstacle (MBDWA) or as a human (HA-WF) the navigation method starts reacting. Therefore it is not necessary to keep the speed of the interactor constant. Nevertheless the interactor attempted to walk with a constant speed.

In the frontal approaching videos (see Figure 1 (b)) the robot controlled by MB-DWA first stops and then moved to the left side. The robot controlled by HA-WF moved smoothly to the right side. In the right and left side approach (see Figure 1 (a) and (c)) the robot controlled by MB-DWA crosses the path in front of the human. The robot controlled by HA-WF stops and lets the human pass its way. The cutting point is defined as the moment when the navigation method starts reacting to the approaching human.

\section{Experimental Conditions}

For our study we used the following two navigation methods: MB-DWA is the move base global planner with the dynamic window local planner; HA-WF is the human aware global planner with the waypoint follower local planner. The MB-DWA method is a state-of-the-art navigation method used by plenty of research groups. We compare the navigation method MB-DWA with the human aware navigation method HA-WF, to find out which concept is the best regarding legibility and perceived safety.

The three different start points for the robot mentioned in Section III-C result in three different approach directions from the human perspective: the robot approaches the human from the left, frontal, or from the right. With three approach directions of the robot and two navigation methods, we tested $3 \times 2=6$ different observation tasks. Each observation task was displayed as a video clip once per participant in random order. To avoid learning effects each video was only displayed once per participant.

\section{E. Experimental Procedure}

First, participants watched four videos to familiarize with the environment and the robot. These introductory videos showed examples of how the robot would move through the room from every direction without the presence of a human and one video of the human walking through the room without the robot. They were presented in a third-person perspective to allow participants to get a feeling for the room. After this the experiment started. We gathered feedback to measure the legibility in each video at two points. First we stopped the video as described in Section III-C. At this point we asked participants to predict the behavior of the robot in the immediate future. Here two categories were provided: velocity (faster, slower, equal, stop) and direction (to one side - right or left, straight forward, stop). After participants marked their prediction, the video was started again and participants were able to observe the complete behavior of the robot. After the robot leaves the camera field of vision, the video was stopped again. Participants were asked to rate whether the robot's actual behavior met their expectations on a 5 point Likert scale $(5=$ very, $1=$ not at all). Additionally we asked for their perceived safety utilizing the respective 
subscale of the Godspeed V questionnaire [4] in which the participant has to rate his/her emotional state regarding anxiety, agitation, and surprise on a five point Likert scale (5 = very, $1=$ not at all) (see also [11]). After the experiment was finished, participants were verbally debriefed. First we asked them to describe their impressions. After this we asked whether they believed that the robot could collide with a person and if they would be afraid of the robot.

\section{DATA ANALYSIS AND RESULTS}

\section{A. Participants}

We recruited 18 participants with the average age of 28 years - thereof 6 women and 12 men. One participant has regular contact with robots, two from time to time and 15 have rarely or no contact with robots.

\section{B. Legibility}

In order to assess the level of legibility of the two navigation methods, participants were asked to predict the robot's behavior at the video break. After watching videos in which the navigation method was HA-WF 29.6\%, after videos with MB-DWA $22.2 \%$ of predictions were correct. Answers were only considered as correct if both direction and velocity were judged correctly by the participant. However, Pearson's ChiSquare test did not reveal a significant association between navigation method and number of correct predictions, $p>.3$. After watching the video to its end, participants had to rate if the robot's behavior matched their expectations. Here we also measured the level of legibility. These ratings were analyzed with a $2 \times 3$ repeated measures ANOVA with the within subject factors navigation method (HA-WF, MB-DWA) and approach direction (left, frontal, right). No significant difference was observed between the navigation methods HA-WF (Mean: $M=2.69)$ and MB-DWA $(M=2.59), p>.7$. However we found a significant main effect for the factor approach direction, $F(2,34)=3.56, p<.05$. Contrasts show that expectations were met to a higher extend when the robot was approaching the person from the front $(M=3.08)$ than when the robot was approaching the human from the left side $(M=2.17), F(1,17)=6.93, p<.05$. No difference was observed between frontal approach or approach from the left and approach from the right $(M=2.67)$, both $p>.09$.

\section{Perceived Safety}

For assessing the perceived safety we asked participants to rate their emotional state regarding the three items anxious, agitated and surprised after watching the videos to their end. According to Bartneck et al. [4], the combination of these measures assesses the perceived safety of a person during interaction with a robot. Note that due to the arrangement of the scale in the Godspeed V questionnaire, a lower rating score stands for a higher perceived safety.

In order to verify possible differences, a $3 \times 2 \times 3$ repeated measures ANOVA with the within subject factors item (anxious, agitated, surprised), navigation method (HAWF, MB-DWA) and approach direction (left, frontal, right) was calculated. It was found that items differ significantly from each other, $F(2,34)=17.43, p<.001$. Contrasts show that the surprise was on average rated higher $(M=2.39)$ compared to the agitation $(M=1.90), F(1,17)=17.81$, $p<01$, and the anxiety $(M=1.68), F(1,17)=33.41$, $p<.001$, with the latter not being different from each other, $p>.1$.

Ratings for navigation method were significantly lower for HA-WF $(M=1.77)$ compared to MB-DWA $(M=2.21)$, $F(1,17)=6.58, p<.05$. As this result is a conglomeration of all measured items, it also provides an impression on how safety was perceived after watching the videos with the different navigation methods. Note again, that due to the arrangement of the scale in the Godspeed $\mathrm{V}$ questionnaire, a lower value indicates a higher perceived safety. Thus, perceived safety was higher for HA-WF than for MB-DWA, see also Fig. 3. Regarding approach direction, no significant differences were observed, $p>.4$. Also no interaction effect was significant, all $p>.2$.

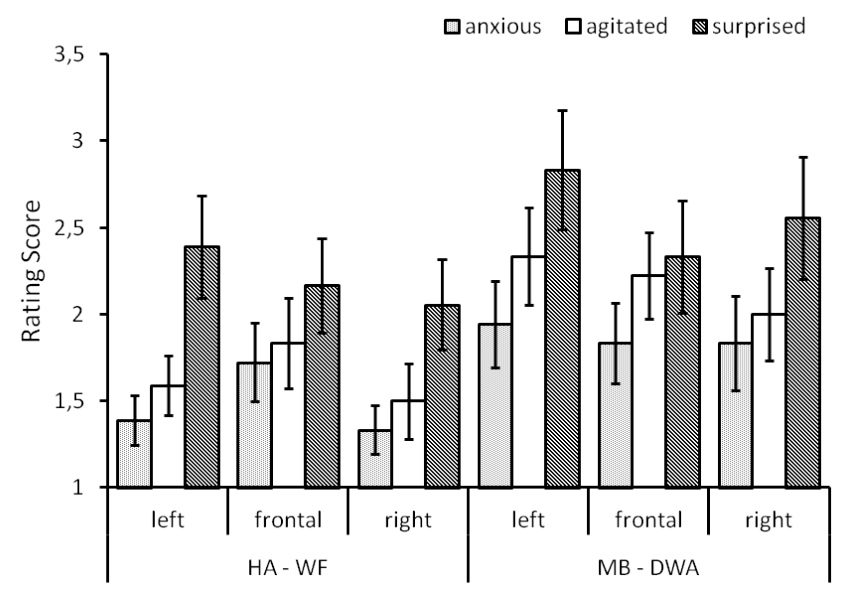

Fig. 3: Godspeed-V-scores of the different items of perceived safety. The perceived safety is higher after watching HA-WF videos. Note: due to the scale in the questionnaire a lower value means higher perceived safety.

\section{Correlation Between Legibility and Perceived Safety}

In order to find out if legibility (measured as correct or incorrect responses) caused a higher perceived safety we performed a correlation analysis, see Fig. 4. For this purpose perceived safety was calculated as the average score of the three items on trial basis. The analysis revealed that there was a significant relationship between the correct answer and perceived safety, $r_{p b}=-.22, p<.05$.

In a next step we also correlated the rating on how much expectations were met with the value obtained for perceived safety. Also here we found a significant relation, $r_{s}=-.29$, $p<.01$, showing that the more expectations were met, the higher the perceived safety was, which is expressed in lower Godspeed-V-scores, see Fig. 5

\section{E. Debriefing}

After the experiment participants were debriefed verbally. No participant was afraid of the robot. Almost all (excluding 


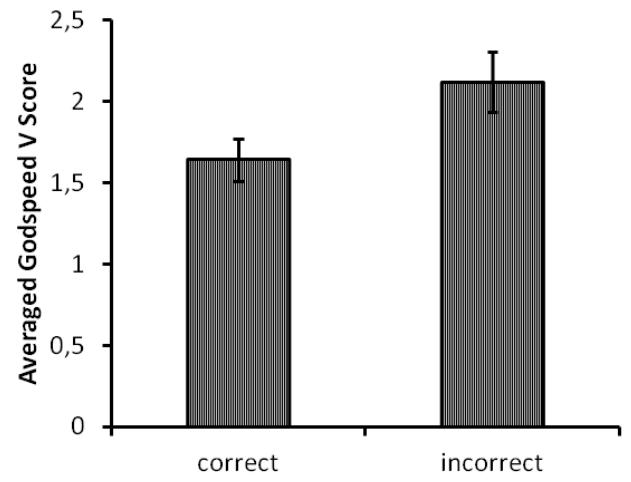

Fig. 4: Relation between correct/incorrect answer and perceived safety. If the answer was incorrect, the average rating score increased which is equivalent with a lower perceived safety.

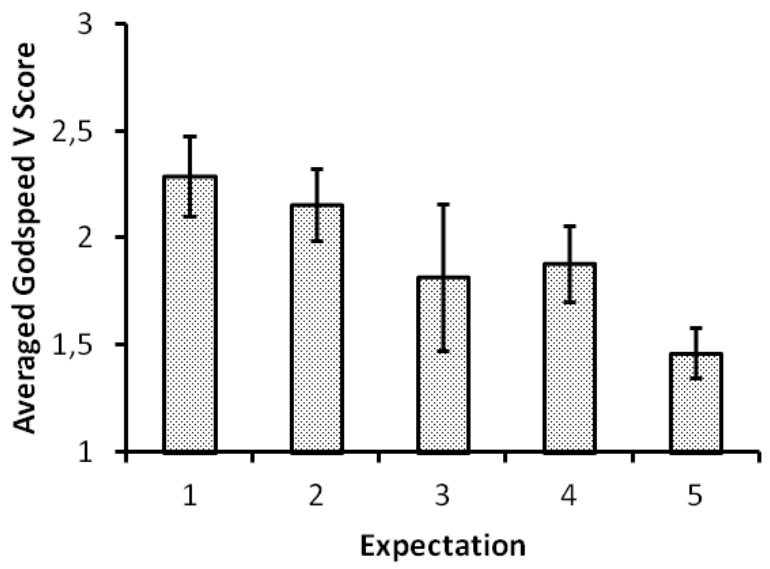

Fig. 5: Relation between perceived safety and expectation score. If expectations are fully met (score $=5$ ), the GodspeedV-score is low, which expresses higher perceived safety.

the one who has regular contact to robots) believed that a robot generally has a built-in safety system which makes sure that the robot never collides with or hurts a human. Most participants (10 of 18) believed that the robot would register them as a human being. Here a frequent comment was: "The robot sees me". To sum up we can say that participants had a basic trust in the robot's safety.

\section{DISCUSSION}

\section{A. Legibility and Perceived Safety}

In our experiment, legibility was measured at two different times. First, participants were asked to predict the robot behavior in the immediate future at the instant the video stopped. However, it is in general problematic to identify the instant of time at which a prediction is made about an approaching robot. It might be that the prediction is made at a different time only after the video was stopped. Therefore we introduced a second judgment time for which participants were able to watch the robot's behavior completely and rated to what extend this observed behavior matched their expectations. Both measures of legibility were analyzed independent of each other. Results show that the HA-WF navigation led to a numerically slightly higher number of correct predictions. Nevertheless there was no significant difference to the responses with MB-DWA navigation, which is not surprising as videos were stopped at that point in time when the algorithm detected human presence and before it was able to react accordingly, see Section III-C. Also no significant differences were observed regarding the legibility of navigation methods after the video was stopped and people had to rate to what extend the robot's behavior matched their expectations. Nevertheless, we found that expectations were met to a higher extent when the robot was approaching the human frontally. This can be explained by looking at the results from the debriefing. It becomes clear that people expect the robot to recognize them. Furthermore, they have a general trust in the fact that the robot would never hurt them, i.e. collide with them. Thus, when the robot is approaching them frontally they expect the robot to react in any way in order not to collide. In this situation, most participants predicted that the robot would stop, which was the correct prediction for the HA-WF. Also many participants predicted that the robot would slow down and turn sidewards, which was not correct for any navigation method.

In general, the number of correct predictions was pretty low. This is also mirrored in the rating of expectations. On average ratings for the two navigation methods are below three, which means that expectations were rather not met with both methods and both resulted in a robot behavior that was not perfectly legible.

However, as no differences were observed regarding the legibility of the navigation methods at both judgment times, it is reasonable to combine the data of the navigation methods for correlation analysis while still keeping the two measures independent. This allows for a general remark on the relation between legibility and perceived safety.

Regarding the latter, we found a significant correlation between legibility and perceived safety for both judgment times. The average Godspeed-V-score obtained after correct responses was lower than after incorrect responses, which means that the perceived safety was higher when people were able to predict the robot's behavior correctly. Regarding the rating of met expectations it was found that the more the expectations were met, the lower the average Godspeed-Vscore was and thus, the higher the perceived safety. Therefore we can assume that the legibility of the robot's behavior is clearly one prerequisite for perceived safety. If the robot's behavior is legible, a person will feel safer during interaction - also if interaction only means crossing paths.

\section{B. Perceived Safety of Navigation Methods}

Although no difference regarding legibility was observed between navigation methods, we found differences regarding the perceived safety. If the robot navigated with HA-WF, a lower Godspeed-V-score was obtained compared to the score obtained after navigation with MB-DWA. Thus, although the human awareness is not the only difference between the 
studied navigation algorithms, our results provide a first hint towards the possibility that if the human is included into the robot's navigation strategy - like the navigation method HA-WF does, the perceived safety on the human side is higher. It is worth mentioning that the perceived safety was in general pretty high (expressed in a low Godspeed-V-score, see Fig. 5). This can first be caused by the experimental situation in which people were only watching a video where the robot is interacting with a person: there was never a real danger of collision with the participant her-/himself. Furthermore the debriefing showed that people have a general trust in the safety mechanisms of the robot. However, if this basic trust would remain during real world interaction is subject to further investigation.

\section{CONCLUSIONS AND FUTURE WORK}

\section{A. Conclusion}

We accomplished an experiment to compare two different robot navigation methods (HA-WF and MB-DWA) regarding their perceived safety and their legibility. The legibility was rather low in both navigation methods with a slightly higher accuracy for HA-WF. A significantly higher perceived safety was measured for HA-WF compared to MB-DWA. Furthermore, we proved our hypothesis that the legibility is correlated with the perceived safety of the robot's behavior.

\section{B. Future Work}

As a next step we will perform a follow-up experiment in a real world scenario where participants are crossing the path of our robot. We expect to measure lower values of perceived safety than in our video based experiment. It remains an open question how legibility, perceived safety, perceived intelligence and acceptance are related. Another open question is if it is possible to assess legibility with objective evaluation measures. To this end we will investigate gaze behavior, pupil size and human motions as factors to determine the legibility of robot behavior. As we consider legibility as an important criterion to evaluate robot behavior, it will be necessary to find further independent and comparable ways of assessing it.

\section{ACKNOWLEDGMENTS}

With the support of the Technische Universität München - Institute for Advanced Study and Cluster for Excellence Cognition for Technical Systems, funded by the German Excellence Initiative and the Bavarian Academy of Sciences and Humanities. Special Thanks to Sandra Hirche for her support, Michael Karg for making the videos and to Thibault Kruse for installing the human aware navigation on the B21 robot.

\section{REFERENCES}

[1] M. Heerink, B. Kröse, V. Evers, and B. Wielinga, "Assessing Acceptance of Assistive Social Agent Technology by Older Adults: the Almere Model,' International Journal of Social Robotics, vol. 2, no. 4, pp. 361-375, Sept. 2010.

[2] V. Venkatesh, M. Morris, G. B. Davis, and F. D. Davis, "User acceptance of information technology: Toward a unified view," MIS Quarterly, vol. 27, no. 3, pp. 425-478, 2003. [Online]. Available: http://www.jstor.org/stable/30036540
[3] A. H. Maslow, "A Theory of Human Motivation," Psychological Review, vol. 50, no. 4, pp. 370-396, 1943. [Online]. Available: http://psychclassics.yorku.ca/Maslow/motivation.htm

[4] C. Bartneck and D. Kulic, "Measuring the anthropomorphism, animacy, likeability, perceived intelligence and perceived safety of robots," Metrics for HRI Workshop, vol. 471, pp. 37-44, 2008.

[5] S. Hudson, J. Fogarty, C. Atkeson, D. Avrahami, J. Forlizzi, S. Kiesler, J. Lee, and J. Yang, "Predicting human interruptibility with sensors: a wizard of oz feasibility study," in Proceedings of the SIGCHI conference on Human factors in computing systems, ser. CHI '03. New York, NY, USA: ACM, 2003, pp. 257-264. [Online]. Available: http://doi.acm.org/10.1145/642611.642657

[6] A. Green, H. Hüttenrauch, and K. S. Eklundh, "Applying the wizardof-oz framework to cooperative service discovery and configuration," in ROMAN 2004 : 13th IEEE International Workshop on Robot and Human Interactive Communication, 2004 : proceedings : September 20-22, 2004, Kurashiki, Okayama Japan at Kurashiki Ivy Square. IEEE, 2004, pp. 575-580.

[7] G. Andersson, K. Höök, D. Mourão, A. Paiva, and M. Costa, "Using a wizard of oz study to inform the design of sentoy," in Proceedings of the 4th conference on Designing interactive systems: processes, practices, methods, and techniques, ser. DIS '02. New York, NY, USA: ACM, 2002, pp. 349-355. [Online]. Available: http://doi.acm.org/10.1145/778712.778762

[8] K. Dautenhahn, M. Walters, S. Woods, K. Koay, C. Nehaniv, A. Sisbot, R. Alami, and T. Siméon, "How may I serve you?: a robot companion approaching a seated person in a helping context," in Proceedings of the 1st ACM SIGCHI/SIGART conference on Human-robot interaction, no. April 2005. ACM, 2006, pp. 172-179. [Online]. Available: http://dl.acm.org/citation.cfm?id=1121272

[9] F. Sadri, "Logic-based approaches to intention recognition," Handbook of Research on Ambient Intelligence: Trends and Perspectives, 2010. [Online]. Available: http://www.igiglobal.com/viewtitlesample.aspx?id=54666

[10] L. Takayama, D. Dooley, and W. Ju, "Expressing thought: improving robot readability with animation principles," in Proceedings of the 6th international conference on Human-robot interaction, ser. HRI ' 11. New York, NY, USA: ACM, 2011, pp. 69-76. [Online]. Available: http://doi.acm.org/10.1145/1957656.1957674

[11] D. Kulic and E. Croft, "Anxiety detection during human-robot interaction," Intelligent Robots and Systems, 2005.(IROS, 2005. [Online]. Available: http://ieeexplore.ieee.org/xpls/abs_all.jsp?arnumber $=1545012$

[12] P. Basili, M. Huber, K. Omiros, T. Lorenz, T. Brandt, S. Hirche, and S. Glasauer, "Inferring the goal of an approaching agent: a humanrobot study," in IEEE International Symposium in Robot and Human Interactive Communication (Ro-Man), 2012.

[13] T. Kruse, P. Basili, S. Glasauer, and A. Kirsch, "Legible Robot Navigation in the Proximity of Moving Humans," in IEEE Workshop on Advanced Robotics and its Social Impacts, 2012.

[14] C. Lichtenthaeler, T. Lorenz, M. Karg, and A. Kirsch, "Increasing Perceived Value Between Human and Robots - Measuring Legibility in Human Aware Navigation," in IEEE Workshop on Advanced Robotics and its Social Impacts, 2012.

[15] E. Marder-Eppstein, E. Berger, T. Foote, B. Gerkey, and K. Konolige, "The office marathon: Robust navigation in an indoor office environment," in Robotics and Automation (ICRA), 2010 IEEE International Conference on. IEEE, 2010, pp. 300-307. [Online]. Available: http://ieeexplore.ieee.org/xpls/abs_all.jsp?arnumber=5509725

[16] D. Fox, W. Burgard, and S. Thrun, "The dynamic window approach to collision avoidance," Robotics \& Automation Magazine, IEEE, vol. 4, no. 1, pp. 23-33, 1997. [Online]. Available: http://onlinelibrary.wiley.com/doi/10.1002/cbdv.200490137/abstract http://ieeexplore.ieee.org/xpls/abs_all.jsp?arnumber $=580977$

[17] B. Gerkey and K. Konolige, "Planning and control in unstructured terrain," in ICRA Workshop on Path Planning on Costmaps, 2008. [Online]. Available: http://pub1.willowgarage.com/apubdb_html/files_upload/8.pdf 